\title{
SPECTRA OF NEARLY HERMITIAN MATRICES
}

\author{
W. KAHAN 1
}

ABSTRACT. When properly ordered, the respective eigenvalues of an $n \times n$ Hermitian matrix $A$ and of a nearby non-Hermitian matrix $A+B$ cannot differ by more than $\left(\log _{2} \dot{n}+2.038\right)\|B\|$; moreover, for all $n \geq 4$, examples $A$ and $B$ exist for which this bound is in excess by at most about a factor 3. This bound is contrasted with other previously published over-estimates that appear to be independent of $n$. Further, a bound is found, for the sum of the squares of respective differences between the eigenvalues, that resembles the Hoffman-Wielandt bound which would be valid if $A+B$ were normal.

0. Our problem. How near are the eigenvalues of a nearly Hermitian matrix to those of a nearby Hermitian matrix? To be specific, let the $n \times n$ matrix $A$ be Hermitian $\left(A^{*}=A\right)$ with eigenvalues $\alpha_{j}$ arranged in ascending order $a_{1} \leq \alpha_{2} \leq \cdots \leq a_{n}$, and let $B$ be an arbitrary $n \times n$ matrix, and index the eigenvalues $\left(\lambda_{j}+\iota \mu_{j}\right)$ of $A+B$ to have real parts $\lambda_{j}$ in ascending order $\lambda_{1} \leq \lambda_{2} \leq \cdots \leq \lambda_{n}$. We seek bounds for differences like $\left|\lambda_{j}+\iota \mu_{j}-a_{j}\right|$ or $\Sigma\left|\lambda_{j}+\iota \mu_{j}-a_{j}\right|^{2}$ in terms of two norms of $B$, one of them

$$
\|B\|_{2} \equiv \sqrt{\operatorname{trace}\left(B^{*} B\right)}=\sqrt{\sum \sum\left|b_{i j}\right|^{2}}
$$

and the other

$$
\|B\| \equiv \max _{z \neq 0} \frac{\|B z\|_{2}}{\|z\|_{2}}
$$

Slightly sharper bounds will be obtained by exploiting the decomposition of $B=X+\imath Y$ into its Hermitian and skew parts

$$
X \equiv\left(B+B^{*}\right) / 2 \text { and } \quad \iota Y \equiv\left(B-B^{*}\right) / 2
$$

Received by the editors January $17,1974$.

AMS (MOS) subject classifications (1970). Primary 15A42, 15A60, 47A55, 65F 15; Secondary 15A18, 15A57, 47A10, 47A30.

Key words and phrases. Non-Hermitian perturbation, eigenvalue error bounds, generalized Hoffman-Wielandt theorem, generalized Weyl inequality.

1 This work was supported in part by a grant from the U. S. Office of Naval Research, contract no. ONR N000 14-69-A-0200-1017. 
whose norms are related to $B^{\prime}$ 's via

$$
\|B\|_{2}^{2}=\|X\|_{2}^{2}+\|Y\|_{2}^{2}, \quad\|X\| \leq\|B\|, \quad\|Y\| \leq\|B\| \leq\|X\|+\|Y\| .
$$

We shall prove that:

(i) Every $\left|\lambda_{j}-a_{j}\right| \leq\|X\|+\|Y\| \cdot\left(\log _{2} n+0.038\right)$ and every $\left|\mu_{j}\right| \leq\|Y\|$, and for every $n \geq 4$ there are matrices $A$ and $B$ for which $X=0$ and the first inequality overestimates some $\left|\lambda_{j}-\alpha_{j}\right|$ by a factor less than 3.

(ii) $\Sigma \mu_{j}^{2} \leq\|Y\|_{2}^{2}$ and $\sqrt{\Sigma\left(\lambda_{j}-\alpha_{j}\right)^{2}} \leq\|X\|_{2}+\sqrt{\|Y\|_{2}^{2}-\Sigma \mu_{j}^{2}}$, and nontrivial equality is possible.

But before these claims are proved in $\$ \$ 2$ and 3 of this paper, here is a survey of what has already been published about our problem.

1. Survey. This survey is drawn from texts like Wilkinson's (1965, pp. 93-109) and Householder's (1964, Chapter 3).

If $A+B$ were normal, the Hoffman-Wielandt theorem (1953) would imply, instead of (ii), $\Sigma\left(\lambda_{j}-a_{j}\right)^{2}+\Sigma \mu_{j}^{2} \leq\|B\|_{2}^{2}$; our weaker hypotheses lead to an inequality weaker by a factor of 2 at worst. If $B$ were Hermitian, the inequalities of H. Weyl (1911, Satz I) would imply (with $X=B, Y=0$ and all $\mu_{j}=0$ ) that $\left|\lambda_{j}-a_{j}\right| \leq\|B\|$, which is a special case of (i) that shows how much may be lost when $Y \neq 0$.

The best bounds pertinent to our problem which I have been able to draw directly from the earlier literature (especially from Wilkinson (1965, PP. 93-94)) involve the congruent truncated disks $D_{j}$ in the complex $(\lambda+\iota \mu)$ plane defined as follows (provided $B \neq 0$ ):

$$
D_{j}=\left\{\lambda+\iota \mu:\left|\lambda+\iota \mu-\alpha_{j}\right|<\|B\| \text { and }|\mu| \leq\|Y\|\right\} \text {. }
$$

Each eigenvalue $\left(\lambda_{k}+\iota \mu_{k}\right)$ of $A+B$ must lie in the closure of that connected component of the union $\bigcup D_{j}$ which includes $D_{k}$. For example, Figure 1 describes a situation with $n=5$ which confines $\left(\lambda_{1}+\iota \mu_{1}\right)$ to $\bar{D}_{1}$, $\left(\lambda_{5}+\iota \mu_{5}\right)$ to $\bar{D}_{5}$, and the remaining three $\left(\lambda_{j}+\iota \mu_{j}\right)$ 's to $\bar{D}_{2} \cup \bar{D}_{3} \cup \bar{D}_{4}$. For another example, consider a situation wherein the $D_{j}^{\prime}$ 's form one long chain in which each $D_{j}$ slightly overlaps its neighbours as shown in Figure 2; without bounds like those proved in this paper there would be no way to explain why all eigenvalues $\left(\lambda_{j}+\iota_{j}\right)$ do not flee like quicksilver to one end of the chain or the other. But (i) above prevents each $\left(\lambda_{k}+\iota \mu_{k}\right)$ from skipping past more than about $1 / 2 \log _{2} n$ of $D_{k}$ 's immediate neighbours, and (ii) above restricts such long skips to at most a small fraction (about $2 /\left(\log _{2} n\right)^{2}$ ) of those $n$ eigenvalues. 
... The $(\lambda+\iota \mu)$-plane...

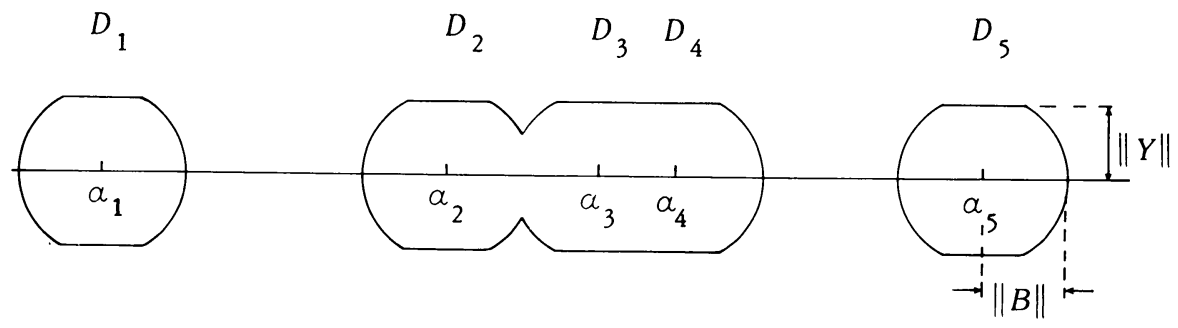

Figure 1. Five eigenvalue estimates

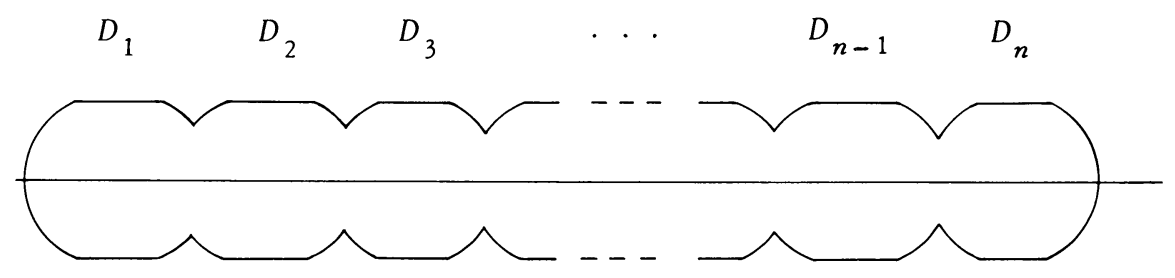

Figure 2. One long chain

2. Proof of claim (i). Our problem is invariant under unitary similarity, so Schur's theorem may be invoked to triangularize $A+B$ by a unitary similarity and then, without loss of generality, we may assume that $A+B$ was given as upper triangular at the outset. Say

$$
A+B=\Lambda+\iota M+\iota U
$$

where $\Lambda \equiv \operatorname{diag}\left(\lambda_{1}, \lambda_{2}, \cdots, \lambda_{n}\right), M \equiv \operatorname{diag}\left(\mu_{1}, \mu_{2}, \cdots, \mu_{n}\right)$ and $U$ is upper triangular with zero for its diagonal. By taking Hermitian and skew parts we find

$$
A+X=\Lambda+\iota\left(U-U^{*}\right) / 2 \text { and } Y=M+\left(U+U^{*}\right) / 2
$$

The last equation will be used below and in $\$ .3$; for now the appropriate bound upon $M$ is found from Bendixson's inequality (cf. Householder (1964, p. 69)) which implies that every $\left|\mu_{j}\right| \leq\left\|(A+B)-(A+B)^{*}\right\| / 2=\|Y\|$. The previous equation is ready for an application of Weyl's in equality; every

$$
\left|\lambda_{j}-a_{j}\right| \leq\|\Lambda-A\|=\left\|X-\iota\left(U-U^{*}\right) / 2\right\| \leq\|X\|+\left\|U-U^{*}\right\| / 2 .
$$

Next set $Z \equiv M+U$ and invoke a theorem published recently (1973) by the author; since $Z$ 's eigenvalues $\mu_{j}$ are all real,

$$
\left\|U-U^{*}\right\|=\left\|Z-Z^{*}\right\| \leq\left\|Z+Z^{*}\right\| \cdot\left(\log _{2} n+0.038\right)=2\|Y\| \cdot\left(\log _{2} n+0.038\right)
$$


which, with the previous inequality, vindicates claim (i) but for the provision of an example.

Take the lower triangular $n \times n$ matrix

$$
L \equiv\left(\begin{array}{ccccccc}
0 & & & & & & \\
1 & 0 & & & & \\
1 / 2 & 1 & 0 & & & \\
1 / 3 & 1 / 2 & 1 & 0 & & & \\
\cdots & \cdots & \ldots & \ldots & \ldots & & \\
\frac{1}{n-2} & \frac{1}{n-3} & \cdots & 1 / 2 & 1 & 0 & \\
\frac{1}{n-1} & \frac{1}{n-2} & \cdots & 1 / 3 & 1 / 2 & 1 & 0
\end{array}\right)
$$

and assemble $A=L+L^{*}$ and $B=L-L^{*}$. In the aforementioned paper (1973) the author demonstrated ${ }^{2}$ that

$$
2 \log n>\|A\|>2 \log n-2 \log 2+1 / 2+1 / n
$$

and that $\|B\|<\pi$. Consequently $A$ has at least one eigenvalue $\alpha_{n} \div 2 \log n$, but all the eigenvalues $\left(\lambda_{j}+\iota_{j}\right)$ of $A+B=2 L$ are zero, so

$$
\left|\lambda_{n}-a_{n}\right|>\|B\| \cdot \frac{2}{\pi}\left(\log n-\log 2+\frac{1}{4}+\frac{1}{2 n}\right) .
$$

Compare this with assertion (i), noting that in this example $X=0$ and $Y=B$ and that $\left(\log _{2} n\right) /((2 / \pi) \log n) \doteqdot 2.27$.

3. Proof of claim (ii). Continuing the analysis in $\$ 2$, observe first that

$$
\begin{aligned}
\|Y\|_{2}^{2} & =\left\|M+\left(U+U^{*}\right) / 2\right\|_{2}^{2} \\
& =\|M\|_{2}^{2}+\frac{1}{2}\|U\|_{2}^{2} \text { (because } M \text { is diagonal and } U \text { above the diagonal) } \\
& \geq\|M\|_{2}^{2}=\sum \mu_{j}^{2}, \quad \text { as claimed in (ii). }
\end{aligned}
$$

2 That paper contains an error due to faulty use of a slide rule. The assertion there that " $(2 / \pi) \log n \doteqdot 0.92 \log _{2} n$ " should read " $\doteqdot\left(\log _{2} n\right) / 2.27$ ". Hence the phrase "about $8 \%$ when $n$ is large" should read "a factor less than 3 when $n \geq 4$ " on pp. 235, 238 and 239. 
Then we find

$$
\begin{aligned}
\sqrt{\sum\left(\lambda_{j}-a_{j}\right)^{2}} & \leq\|\Lambda-A\|_{2} \quad \text { (by the Hoffman-Wielandt theorem) } \\
& =\left\|X-\iota\left(U-U^{*}\right) / 2\right\|_{2} \leq\|X\|_{2}+\left\|(U-U)^{*} / 2\right\|_{2} \\
& =\|X\|_{2}+\|U\|_{2} / \sqrt{ } 2=\|X\|_{2}+\sqrt{\|Y\|_{2}^{2}-\sum \mu_{j}^{2}} \text { as claimed in (ii). }
\end{aligned}
$$

These in equalities reduce to something slightly stronger than the HoffmanWielandt theorem when $A+B$ is normal because then $U=0$ so $\Sigma \mu_{j}^{2}=\|Y\|_{2}^{2}$ and, hence, $\Sigma\left(\lambda_{j}-\alpha_{j}\right)^{2} \leq\|X\|_{2}^{2}$; whereas the Hoffman-Wielandt theorem in its raw form would imply only that the sum $\Sigma \mu_{j}^{2}+\Sigma\left(\lambda_{j}-a_{j}\right)^{2} \leq\|X\|_{2}^{2}+\|Y\|_{2}^{2}=$ $\|B\|_{2}^{2}$. On the other hand, if we do not know separate bounds for $\|X\|_{2}$ and $\|Y\|_{2}$, but only one bound for $\|B\|_{2}$, we can still exploit (ii) as follows:

$$
\begin{aligned}
\sum\left(\lambda_{j}-\alpha_{j}\right)^{2}+2 \sum \mu_{j}^{2} & \leq\left(\|X\|_{2}+\sqrt{\|Y\|_{2}^{2}-\sum \mu_{j}^{2}}\right)^{2}+2 \sum \mu_{j}^{2} \\
& =2\|X\|_{2}^{2}+2\|Y\|_{2}^{2}-\left(\|X\|_{2}-\sqrt{\|Y\|_{2}^{2}-\sum \mu_{j}^{2}}\right)^{2} \\
& \leq 2\left(\|X\|_{2}^{2}+\|Y\|_{2}^{2}\right)=2\|B\|_{2}^{2} .
\end{aligned}
$$

Although the last inequality is not as tight as that in (ii), both in equalities can be made nontrivial equalities by an example:

$$
\begin{gathered}
A \equiv\left(\begin{array}{cc}
0 & 1 \\
1 & 0
\end{array}\right), \quad \alpha_{1}=-1, \quad \alpha_{2}=1 ; \\
B \equiv\left(\begin{array}{cc}
\iota \mu_{1} & 0 \\
-1 & \iota \mu_{2}
\end{array}\right), \quad\|X\|_{2}^{2}=1 / 2, \quad\|Y\|_{2}^{2}=1 / 2+\mu_{1}^{2}+\mu_{2}^{2}, \quad\|B\|_{2}^{2}=1+\mu_{1}^{2}+\mu_{2}^{2} ; \\
A+B=\left(\begin{array}{cc}
\iota \mu_{1} & 1 \\
0 & \iota \mu_{2}
\end{array}\right), \quad \lambda_{1}=\lambda_{2}=0,
\end{gathered}
$$

and

$$
\sqrt{ } 2=\sqrt{\sum\left(\lambda_{j}-\alpha_{j}\right)^{2}}=\|X\|_{2}+\sqrt{\|Y\|_{2}^{2}-\sum \mu_{j}^{2}},
$$

and finally

$$
\sum\left(\lambda_{j}-\alpha_{j}\right)^{2}+2 \sum \mu_{j}^{2}=2\|B\|_{2}^{2} .
$$

4. Caveat. Sometimes our problem of $\S 0$ comes with the additional information that all of $(A+B)$ 's eigenvalues are real. By itself this informa- 
tion confers little advantage for the estimation of $\max _{j}\left|\lambda_{j}-\alpha_{j}\right|$ or $\Sigma\left(\lambda_{j}-a_{j}\right)^{2}$ beyond what is already available from (i) and (ii), as we see from the two examples $A+B$ given above; both examples can have all eigenvalues real (i.e. zero).

But whence comes the knowledge that all of $(A+B)^{\prime}$ s eigenvalues are real? Frequently this is inferred from the existence of a positive definite Hermitian matrix $H$ for which $A+B=H A$. If bounds are known for $\|H\|$ and $\left\|H^{-1}\right\|$, then the eigenvalues $\lambda_{j}$ of $A+B$ compare with the eigenvalues $\alpha_{j}$ of $A$ as follows (cf. Weyl (1912, Satz IV)); for each $j$ either $1 /\left\|H^{-1}\right\| \leq \lambda_{j} / \alpha_{j}$ $\leq\|H\|$ or $\lambda_{j}=a_{j}=0$. These inequalities, if available, are generally sharper than the ones proved earlier in this paper.

Less often we may know that $V \equiv F(A+B) F^{-1}$ is Hermitian for some similarity $F$ whose condition number $\kappa \equiv\|F\| \cdot\left\|F^{-1}\right\|$ is known not to be large. In this case we may prove all $\left|\lambda_{j}-\alpha_{j}\right| \leq \kappa \cdot\|B\|$, which is better than (i) whenever $\kappa<\log _{2} n$, and also a sharper bound than Wilkinson's (1965, pp. 87-88) whenever the bounds for two different $\lambda_{j}$ 's overlap. The following proof of the foregoing inequalities is adapted from an unpublished earlier report by the author (1967).

The polar factorization $F=Q H$ provides a Hermitian positive definite $H \equiv\left(F^{*} F\right)^{1 / 2}$ with $\|H\|=\|F\|$ and $\left\|H^{-1}\right\|=\left\|F^{-1}\right\|$, as well as a unitary $Q$, and $Y \equiv Q^{*} V Q$ has the same eigenvalues $\lambda_{j}$ as have $V$ and $A+B$. Weyl's inequalities will yield the desired result $\left|\lambda_{j}-\alpha_{j}\right| \leq \kappa \cdot\|B\|$ if we can prove $\|Y-A\| \leq \kappa \cdot\|B\|$. But first let $x$ be a normalized $\left(x^{*} x=1\right)$ eigenvector of $Y-A$ for which $(Y-A) x= \pm\|Y-A\| x$. Then

$$
\begin{aligned}
\|F\| \cdot\|B\| & =\|H\| \cdot\left\|F^{-1} V F-A\right\|=\|H\| \cdot\left\|H^{-1}(Y H-H A)\right\| \geq\|Y H-H A\| \\
& \geq\left|x^{*}(Y H-H A) x\right|=\left|x^{*}(Y H-H Y) x+x^{*} H(Y-A) x\right| \\
& =\left|x^{*}(Y H-H Y) x \pm\|Y-A\| x^{*} H x\right|=\mid \text { imaginary } \pm \text { real } \mid \\
& \geq\|Y-A\| x^{*} H x \geq\|Y-A\| /\left\|H^{-1}\right\|=\|Y-A\| /\left\|F^{-1}\right\| .
\end{aligned}
$$

\section{REFERENCES}

A. S. Householder (1964), The theory of matrices in numerical analysis, Blaisdell, New York. MR $30 \# 5475$.

A. J. Hoffman and H. W. Wielandt (1953), The variation of the spectrum of a normal matrix, Duke Math. J. 20, 37-39. MR 14, 611.

W. Kahan (1967), Inclusion theorems for clusters of eigenvalues of Hermitian matrices, Computer Science Department, University of Toronto, Toronto, Ontario. 
W. Kahan (1973), Every $n \times n$ matrix $Z$ with real spectrum satisfies $\left\|Z-Z^{*}\right\|$ $\leq\left\|Z+Z^{*}\right\|\left(\log _{2} n+0.038\right)$, Proc. Amer. Math. Soc. 39, 235-241. MR $47 \# 1833$. H. Weyl (1911), Das asymptotische Verteilungsgesetz der Eigenwerte linearer partieller Differentialgleichunger,..., Math. Ann. 71, 441-479.

J. H. Wilkinson (1965), The algebraic eigenvalue problem, Clarendon Press, Oxford. MR $32 \# 1894$.

ELECTRONICS RESEARCH LABORATORY, UNIVERSITY OF CALIFORNIA, BERKELEY, CALIFORNIA 94720

Current address: Department of Mathematics, University of California, Berkeley, California 94720 